\title{
Platforms for Online Learning: A Product Specification
}

\section{Mtro. Gerardo Quiroz Vieyra}

Professor of the Department of Politics and Culture of the Universidad Autónoma Metropolitana (UAM) Xochimilco Unit. Communications and Electronics Engineer from the National Polytechnic Institute, Master in Administration and Consulting from the Marista University. Former Director of Informatics of the UAM, Former Secretary of Unit of the UAM Cuajimalpa, Former Secretary of Strategic Management of the IPN. E-mail:

\section{Dr. Luis Fernando Muñoz González}

Professor of the Department of Economic Production of the Universidad Autónoma Metropolitana (UAM) Xochimilco Unit. Degree in Administration and Degree in Economics from the UAM, Master in Finance from the Chapultepec University and Doctor in Social and Administrative Sciences from the Chapultepec University.

\begin{abstract}
Learning Management Systems (LMS) or Learning Content Management Systems (LCMS) are the core of e-learning platforms and have evolved according to the development of new information and communication technologies. In this type of software there are many products on the market, some developed by the institutions themselves (in-house), others are free and open source software (FOSS) and others are more commercial, varying in functionality and technology, but almost always adhering to the standards used in e-learning so that learning objects fulfill their purpose of being usable and reusable. This paper introduces you to current LMS / LCMS, main functions, distinctive features, related standards, and their current status. Then there is a presentation of Machine Learning as a branch of Artificial Intelligence and Cognitive Computing as a fusion discipline between computation, cognition, psychology and artificial intelligence, to end in a proposal for the incorporation of these technologies in a new generation of e-learning platforms, all in an integrated framework of interoperability and governance.
\end{abstract}

Keywords: Cognitive computing, learning content management system, learning management systems, learning objects, machine learning.

\section{Introduction}

Computerized systems for learning have a history dating back to the last century, for example, PLATO (Programmed Logic Automated Teaching Operations) was developed in 1960 at the University of Illinois at Urbana-Champaing and was one of the first generalized computerized assistive systems. Having systems like PLATO by origin, learning and training have adopted these technologies as support, as a complement and even as an environment for their activities, with all the pros and cons that can be had. 
Modern e-learning is based on electronic platforms, its core is learning management systems (LMS). These systems, present for almost two decades, have been evolving in characteristics and functionality by incorporating advances in information and communication technologies and design, moving from material repositories with access control, to platforms with administration and analysis capabilities. . At the same time, other types of applications that gravitate to the learning environment have also been created and developed, such as administrative systems and social networks, for example, that communicate through interfaces with it, to give them functionality or information about the environment that is not available in them, giving rise to the application of classical learning theories and also those of the 21st century (Harasim, 2017), such as connectivist and collaborative.

Also, and in the past decade, other technologies that were not possible before due to the high computing capacity necessary for their instrumentation, such as Big Data, advanced analytics (Big Data analytics) and artificial intelligence instrumentation that the century in the past they had been conceptualized but remained in written projects or as laboratory prototypes.

The massification of Artificial Intelligence (AI), the simulation of human intelligence through computers, was announced for 1995 under the flag of Japan, which did not happen, but AI has arrived, first silently through its incorporation into various products such as anti-spam mail filters, vision in robots and advanced video surveillance systems, and to a very limited extent in some dictation software, but now there is a huge wave of AI applications, found in all types of computer and communications, from smart cell phones to supercomputers, and for all kinds of applications: health, production, logistics, administration, education, agriculture, and even in the configuration, monitoring, operation and support of the computer and communications systems themselves.

These new technologies are not only possible in all types of computer equipment, but are also now accessible to those who have the knowledge to use them, since there is software for artificial intelligence both FOSS (Free Open Source Software), as well as freemium and commercial. This opens up an immense possibility of using AI in all kinds of applications, including e-learning platforms.

\section{Methodological aspects}

\section{E-learning platforms}

From an information and communication technology (ICT) point of view, a platform is made up of the hardware, the operating system, and the specific purpose application in question. So, when talking about platforms, it is implicitly referring to the integration of at least these three elements, which is important because the total computing capacity will be determined by them.

Michael Piotrowski (2010) gives the following definition: "An e-learnig plataform is a system which provides integrated Support for the six activities -creation, organization, delivery, communication, collaboration, and assessment- in an educational context". Thus, an e-learning platform can be seen as a managed repository of educational elements, the learning objects.

Currently two are the predominant types of systems for e-learning: LMS (Learning Management Systems) and LCMS (Learning Content Management Systems), which can also be divided into academic and corporate. The LMS are based on repositories of digital content, called learning objects, which resemble libraries where the volumes are electronic files in 
different formats, those supported by the e-learning standard that the platform supports, in addition these platforms have creation functions of courses and trajectories, application and qualification of exams, exam simulators, user registration and more. LCMS, although the term is currently used as a synonym for LMS, can be seen as a version with greater functionality than an LMS, since they not only have the functions of an LMS, but also include the entire cycle of creation, publication, delivery and measurement of content usage,

What distinguishes this type of platform from other types of information systems is its instructional content, while others store data, information or knowledge, these systems store information and knowledge in the form of learning objects, that is, capsules of necessary content and sufficient for the achievement of an educational objective. This feature requires special types of storage in order to support interoperability and authoring tools.

The main standards used in the content of e-learning systems are AICC, SCORM, xAPI and cm5. The oldest standard is AICC (Aviation Industry Computer-Based Training Committee) which uses the HTTP protocol, and although it has not evolved some LMS and some authoring tools still use it. The de facto standard is SCORM (Sharable Content Object Reference Model) of which there are four versions, of which two are currently in use, 1.2 and 2004. A newer standard that allows user "experiences" to be recorded, online or offline online, and that has capabilities for mobile learning, social learning, offline learning, and collaborative learning is xAPI. Even more recent is cm5, launched in 2016, which brings together the benefits of SCORM and XAPI in a single standard, which means that content created under this standard can be used on mobile devices and even offline. It is worth mentioning that, although SCORM is the most popular standard, due to its limitations some platforms such as Moodle, although they plan to continue supporting SCORM, will make their new developments in XAPI.

Steven D. Foreman (2018), makes a good description of this type of systems and the various aspects to consider in their selection, implementation and operation, as well as in their possible operation with other repositories, but above all tries to give a simple vision of what constitutes an LMS / LCMS, however these aspects correspond to the technological platform, but that what truly gives value to the platform is its content, the instructional material in the form of learning objects, which must be planned and built based on the instructional design (Muñoz and Quiroz, 2019).

In this type of software, LMS / LCMS, there are a large number of products, some are their own developments (SEND by UAM Xochimilco, for example), others of the FOSS type (Moddle and Canvas as the most representative) and many others of the commercial (like BlackBoard and Docebo to mention two of the most popular). It really is a very competitive sector, in which there are two main types, for academic use and for business use, although some are used in both cases, such as Moodle.

Klaus Schwab (2016) argues that platforms are dominating the dynamics of productive and commercial activities due to their cost-benefit relationship, since the increase in operations only marginally increases their operating cost. This is applicable to e-learning platforms, an increase in the number of enrolled and active students does not increase the operating cost of the platform in the same proportion, remembering that this cost is not only the cost of the hardware, but also that of the software and support staff. 


\section{Comprehensive platforms}

Although LMS / LCMS systems have repositories of learning objects in various formats (text, audio, video, etc.), it should not be overlooked that many other contents can even be found on other platforms. Quiroz and Muñoz (2018a) in a systemic approach to e-learning that starts from a comprehensive ecosystem, also propose a comprehensive e-learning platform (Quiroz \& Muñoz, 2018b) that having an LMS / LCMS as a core is complemented with other resources technologies, both with academic content and applications as well as with analytical tools that give or expand this capacity in the LMS / LCMS, all in a systems governance environment, because there must be policies, criteria and rules to integrate and operate the systems in order to to avoid chaotic operation, poor performance or neglect of one or any of them.

It must be taken into account, however, that there are interoperability issues between systems and applications, because communication between them must be ensured; as well as continuity in the operation, because it is necessary to ensure that the system works most of the time (for example, $99.99 \%$ of the time, which is the expected figure in a business-class information system), and for this, that Measures are in place for the continuity of operation, either with redundant systems or with a backup and recovery system that in the event of an equipment failure or an external attack allows the content to be recovered without much loss due to the time elapsed between the backup and its recovery. Also, that this platform has protection systems against cyber attacks, such as intrusions and malware.

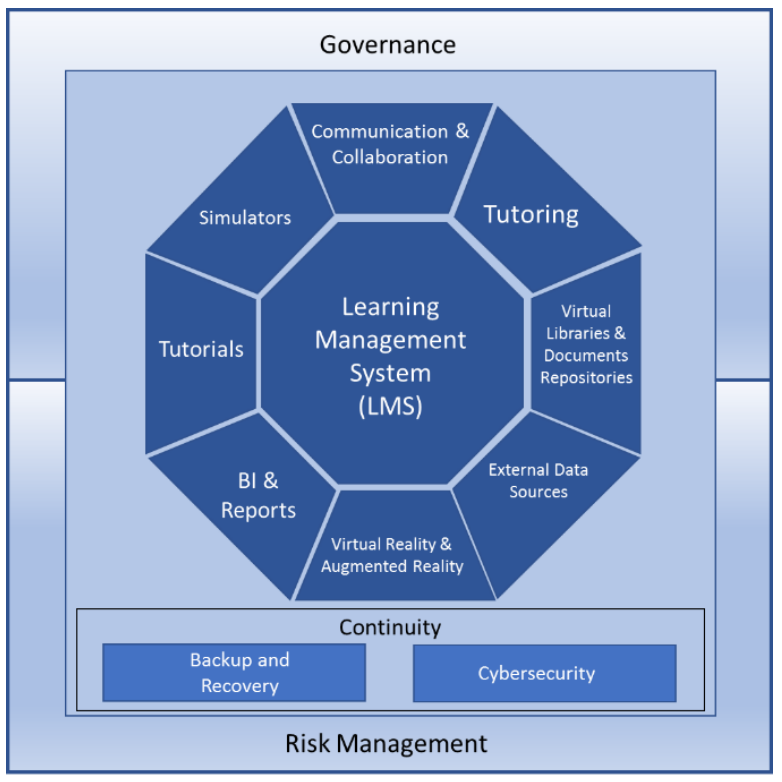

Figure 1. Comprehensive e-learning platform

Source: Quiroz \& Muñoz (2018b)

The most advanced LMS / LCMS have the ability to incorporate the use of external repositories through communication protocols such as LTI (Learning Tools Interoperability) and there are proposals for comprehensive platforms, called XXX, but we must not forget that they should 
be considered as any another type of information system, with the same rigor in the selection, implementation, operation, maintenance and security criteria.

\section{Machine Learning}

The Machine Lerning (ML), also known as machine learning, is one of the branches of artificial intelligence, which fundamentally consists of the analysis of data to identify relationships, patterns and groups, and as Sergios Theodoridis (2020) says: "Machine Learning is a name that is gaining popularity as an umbrella for methods that have been studied and developed for many decades in different scientific communities and under different names, such as Statistical Learning, Statistical Signal Processing, Patter Recognitions..., and Computational Learning. The name "machine learning" indicates what all these disciplines have in common, that is, to learn from data, and then make predictions".

Thus, machine learning is a collection of methods that can be used not only to analyze data, but to learn from them and use that knowledge in many activities that would ordinarily be done manually, for example, in the diagnosis and configuration of data networks, database administration, mail filtering, fraud detection, recommendation systems (in e-commerce), etc. As can be seen, the use of machine learning is very broad, and of course it also has applicability in education, in any of its modalities: face-to-face, virtual or hybrid, both in the academic part and in the administration part.

Machine Learning makes use of three types of mathematical methods: regression, classification, and clustering. David Forsyth (2019) said that: "Regression accepts a feature vector and produce a prediction, which is usually a number, but can sometimes have an other forms. You can use these predictions as predictions, or to study trends in data...", also said: "A classifier is a procedure that accepts a set of features and produce a label. Classifiers are trained on labelled examples, but the goal is to get a classifier that performs well on data, which is not seen at the time of training. Training a classifier requires labelled data that is representative of future data". Clustering is the task of grouping similar objects and is used to find structures in data that are not obvious to the naked eye.

The mathematical methods used in machine learning are probabilistic or statistical instrumented through algorithms, and among the most used are Bayesian classifier, nearestneighbor, decision trees, Support Vector Machine, random forest, principal component analysis (PCA), canonical correlation analysis, k-means, linear regression, logistic regression, hidden Markov models, dynamic programming, nearest neighbor, and many others, almost all of them based on the measurement of variability, correlation and closeness between the elements to be processed, and be it data, sounds or images, that is to say in structured or unstructured data.

There are three types of machine learning: supervised, in which the system is trained with sample data to recognize the expected results, in this type decision trees, regression algorithms, K-Nearest Neighbors and random forest are frequently used; non-supervised, is one in which learning occurs from the analysis of data without an expected response, but rather the result obtained from the different inputs is analyzed, for this type of learning the most common methods are clustering algorithms, neural networks and Markov models; The third type, reinforcement machine learning, is one in which the results obtained from the methods used are qualified to establish valid learning, mainly using hidden Markov models and dynamic programming. 


\section{Cognitive computation}

Cognitive computing is interdisciplinary, it combines cognitive science, the science of the mind, and computational science, the science and technique of the design and application of computational systems. Cognitive computing is a new era of computing, it is the third evolution of computers, the first being electromechanical tabulating machines built to do a single task; the second, programmatic machines, that is, general-purpose machines that can be programmed for some task according to John Von Newmann's concept of stored program; and the third, cognitive computing systems, designed to extend the limits of human cognitive capacity and interact in a natural way with humans. It is not visualized that the second generation disappears, but is complemented with the products of the third, so that more and more computer products with cognitive capabilities will be seen, that is, the incorporation of cognitive computing functions in the information systems developed based on explicit programming.

Researchers from IBM consider that cognitive computation and human capacities are complementary (Alfio Gliozzo, et al., 2017, PP. 6), because while the human being is characterized by having common sense, understanding, imagination, morality, abstraction, generalization and reverie, cognitive computing is useful for locating knowledge, identifying patterns, natural language management, machine learning, elimination of biases and a work capacity only limited by the capacity of the computing system in which it is executed. It should be noted that cognitive computing includes machine learning, complemented with other technologies that facilitate its interaction with the environment, such as image recognition and natural language management, as mentioned before.

John E. Kelly III (2015) says that "Cognitive computing (CC) refers to systems that learn at scale, reason with purpose and interact with humans naturally. Rather than being explicitly programmed, they learn and reason from their interactions with us and from their experiences with their environment." Cognitive computing systems follow a decision process similar to that of humans: (1) they observe the phenomena, (2) they interpret the evidence and generate a hypothesis, (3) they evaluate the hypothesis and (4) they decide by selecting the one they consider the best option and act; all on structured and unstructured data.

As cognitive computing is an area of great interest for scientific, technological and commercial development, there are many companies developing products for this field. IBM, a pioneer in research in cognitive computing, designed a cognitive processor (IBM, 2011) and its IBM Watson system is widely known for its successful implementation for diagnosing and recommending cancer treatments. Other large companies such as Microsoft, Google, Amazon Web Services, CISCO, Tata Computing Services and SAS, among many others, have also developed software or hardware for this type of system. Google, for example, has already developed three generations of TPUs (Tensor Process Units, specific purpose processors for the execution of tensioner algorithms) and the most advanced smartphone processors no longer only include CPUs and GPUs, but also neural networks. , applicable to machine learning and deep learning. This means that products for cognitive computing will be increasingly available and accessible, and therefore can be used in a greater number of applications, including education. 


\section{Discussion: Towards an intelligent platform}

Existing machine learning and cognitive computing technologies can be applied to e-learning platforms to equip them with intelligence in the form of higher analytics, learning, and reasoning.

Some LMS are beginning to incorporate machine learning algorithms to analyze the data recorded by the use of different resources by students, based on them, groups of like-minded students are created, but their use may be greater than this. Applying machine learning to the data of the activities of the students could also identify their individual progress patterns to predictively establish the possible problems in their performance. Additionally, machine learning can be applied to the instructional content of the system to analyze the learning objects and establish, for example, the relevance in the predecessors and the appropriate successor sequence, as well as evaluate their quality.

Regression algorithms - for example, logistic regression could be applied to predict student performance based on variables such as the school of origin, their learning profile, performance in previous cycles, etc. Those of classification to create trees with probabilities in the different sequences of courses and contents. And those of clustering to establish groups between students, but also between learning objects, among other things to identify exceptions and seek integration between them.

These are just some of the possible applications of machine learning in e-learning platforms, although they could also be integrated as part of the platform's ecosystem in its educational modules, in the form of an advanced analytical tool for student learning activities.

In a second generation of intelligent e-learning platforms, incorporating cognitive computing, the systems would have the ability to analyze student data, formulate hypotheses about their trajectory and school performance, make deductions and propose personalized sequences of learning objects and even of topics, which would lead to an approach to trajectories and personalized curricula by the system, which could initially be validated by the teachers, as long as the adjustment in the training of these systems lasts.

Applied to learning objects, they could be analyzed at the content level to determine a usage and content quality rating based on the criteria established, in order to determine the possible need to adjust and replace them. Likewise, with the ability to understand natural language and making use of the content of the learning objects, boots could be built to attend to the basic queries of the students and turn the teacher over only those that they cannot attend, in addition to the platform itself in a complement in the teaching-learning process by becoming expert systems and simulation systems.

These analytical capabilities, through machine learning or cognitive computing (which includes machine learning) of diagnosis and proposal, would far exceed the dashboards and reports that they currently generate.

Conclusions and recommendations:

So far, e-learning platforms have followed the strategy of incorporating the advances and trends of information and communication technologies, which has also resulted in the possibility of aligning them with instructional design and learning objects, but fundamentally establishing themselves as repositories with capacities for evaluating students and administering courses and other learning activities, with capacities for performance indicator 
boards and reports very much in line with BI (Business Intelligence) systems, and with limited use of machine learning.

Although depending on the technological, financial and institutional resources, both machine learning and cognitive computing are technologies that are already available and that therefore would be applicable in education, both in the teaching-learning process and in platforms e-learning themselves. The use of FOSS-type software could be a very good spearhead for this because for small projects, in addition to not requiring very powerful computer equipment and costs, the software itself can be used at no cost, such as the aforementioned TensorFlow and Keras for deep learning and natural language processing, and machine learning libraries for $\mathrm{R}$ and Python. Naturally there is a learning curve for this software, but the approach to the models and the mastery of mathematical methods would be even more challenging, although the reward would be very high, the discovery of the knowledge stored in the data.

When including ML or CC, either as part of the platform or as satellite systems to it, the interoperability, performance and governance issues mentioned above for end-to-end platforms must also be considered, as they will naturally require greater computing power and storage than current platforms, and the complexity of administration and operation will also increase, but with an added value that can be the trigger for a much more effective and efficient use of LMS / LCMS platforms.

\section{References}

[1] Foreman, S. (2018). The LMS Guidebook: Learning Management Systems Demystified. ATD Press, Alexandria, VA

[2] Forsyth, D. (2019). Applied Machine Learning. Springer Nature Switzerland AG, Cham, Switzerland. https:/doi.org/10.1007/978-3-030-18114-7-1

[3] Gliozzo, A, Ackerson, Ch., Bhattacharya, R., Goering, A., Jumba, A., Yeon Kim, S., Krishnamurthy, L., Lam, T., Littera, A., McIntosh lain, Murty, S., Ribas, M. (2017). Building Cognitive Applications with IBM Watson Services: Volume 1, Getting Started. International Business Corporation, USA.

[4] Harasim, L. (2017). Learning Theopry and Online Technologies. 2nd. Edition. Routledge, New York, NY.

[5] Muñoz González, L., Quiroz Vieyra, G. (2019). Instructional Design in Online Education: a Systemic Approach. European Journal of Education. 2(3):64. DOI: 10.26417/ejed.v2i3.p64-73

[6] Quiroz G., Muñoz, L. F. (2018a). Creación de Capital Intelectual para el Aprendizaje Electrónico en las Universidades Públicas Mexicanas. XXXI Simposio Internacional de TIC en la Educación SOMECE 2018, CDMX, México.

[7] Quiroz G., Muñoz, L. F. (2018b). Una arquitectura integrada de tecnologías digitales para la educación a distancia. X Congreso Ibero-Americano de Docencia Universitaria, Porto Alegre, $\begin{array}{llll}\text { Brasil. } & \text { Retrieved 16, 2020: }\end{array}$ https://ebooks.pucrs.br/edipucrs/acessolivre/anais/cidu/assets/edicoes/2018/comp-listdocs.html?\#

[8] Piotrowski, M. (2010). What is an E-Learning Platform? In Kats, Y. Learning Management System Technologies and Software Solutions for Online Teaching: Tools and Applications. (pp 31). Information Science Reference (an Imprint of IGI Global), Hersey, PA.

[9] Kelly, J. III (2015). Computing, cognition and the future of knowing: How humans and machines are forging a new age of understanding. IBM Research, Albany, NY. Retrieved November 9, 2020: https://www.research.ibm.com/software/IBMResearch/multimedia/Computing_Cognition_WhitePa per.pdf 
[10] Schwab, K (2016). The Fourth Industrial Revolution. World Economic Forum, Cologny/Geneva, Switzerland.

[11] Theodoridis, S. (2020). Machine Learning. A Bayesian and Optimization Perspective. 2nd. Edition. Academic Press, Waltham, MA. 